\title{
Information technology supporting daily activities of seniors
}

\author{
Dušan Šimšík *, Alena Galajdová, Daniel Siman, Marianna Andrášová, and Róbert \\ Balog
}

\author{
Department of Automation, Control and Human Machine Interactions, Technical University \\ of Košice, Košice, Slovakia \\ *Corresponding author (dusan.simsik@tuke.sk)
}

\begin{abstract}
Purpose Currently, ICT-products play an important role in improving the quality of life and matching user needs in the information society. The ICTs and embedded tools in public services improve users' autonomy, safety, self-realization, social inclusion by facilitating communication between family members, carers and persons with disabilities, etc. Our research team participated in the 6FP-project called MonAMI1 devoted to the development of accessible and usable services for seniors that can be delivered in mainstream systems and platforms. Method The MonAMI technology platform was derived from standard technologies and has a unique architecture based on the Residential Gateway (RG-PC with touchscreen), OSGi-platform2 (the dynamic module system and service platform for JavaTM), sensors and actuators dedicated to monitoring and control of the senior's households. Sensors and actuators use open technology networking such as wireless ZigBee combined with wired 1-wire nodes. MonAMI-system and services provide local and remote monitoring, local and remote actuating, detection and alarm notifications of potential dangerous situations. Monitoring services monitor various household's parameters. Data about room temperature, luminosity, motion detection, usage of electrical appliances are sent automatically to the RG and processed in real time. In case of unusual behavior detection, the alarm is triggered to alert the responsible person, e.g. the carer. Light, shutter, and electrical appliances actuators controlled automatically or by individual depending on setting are used to facilitate seniors' everyday life. Results \& Discussion The technology and developed services were firstly tested in a laboratory environment with perspective users and their carers. Later, 20 participants living alone in their households were chosen for a three-month trial where different experimental testing was running under real conditions. We obtained information about usefulness of each service and device, advantages and deficiencies of the system, participant's feelings about new technologies and so on. The system has helped the majority of participants in terms of increased safety. Carers confirmed the unloading of the responsibility affected their daily life. Results were used for the design set-up of the new research project; at the same time we continued supporting the municipality by running MonAMl-services for the test group of seniors in the MonAMI-project after completion.
\end{abstract}

Keywords: ICT, monitoring, automation, household, elderly

\section{INTRODUCTION}

As the ICT is growing rapidly and it is becoming an integral part of daily life e.g. in the way of communication, obtaining of information, data handling, etc., there is a potential of increasing social exclusion. The most vulnerable are groups of elderly people and people with disabilities.because of their economy and health status. They are exposed to the risky situation of excluding from use of new technologies due to the low availability, accessibility or literacy of ICTs usage. Services and technologies based on ICTs designed for elderly and people with disabilities offered as a mainstream products are insufficient. One from our objectives has been to demonstrate that services and technology platform derived from standard technologies, developed in MonAMI project, is accepted by elderly and they are supportive in their daily activities.

\section{DESCRIPTION OF SYSTEM - MONAMI PLATFORM}

The idea of MonAMI platform was to construct technology platform which is derived from sub-systems of standard technologies. The platform is assigned to deliver services, which integrate reliable wireless (ZigBee technology), Self Organizing Networks, wired networks (1-wire technology), user friendly devices for interaction, wearable devices and components for health monitoring. 1

Sensors and actuators are connected to the Residential Gateway, where the main logic of the system is running basically dedicated for evaluation and determination of next action. Due to the usage of OSGi4AMI hardware implementation of the sensors and actuators is transparent to the services. They appear in the system as software objects with predefined methods and functionality.

Residential Gateway could be any computer with x86 architecture and Java ${ }^{\mathrm{TM}}$ technology support. Its 
role is to centralize the MonAMI services in one point which enables services to share the same resources and afford opportunity to create extended services. The user can interact with MonAMI system via intuitive user interface (UI). In our project and trials we have used as the RG Asus EeeTop - touch screen computer. The UI may, however, run remotely on another ICT device such as PDA, smart phone, tablet, another computer. The communication between $R G$ and user interface is established with predefined protocol. We have used $\mathrm{UCH}$ protocol which is based on HTTP and socket communication. The Universal Control Hub was developed within the scope of the 6FP project i2home and is provided by the Universal Remote Console Consortium. ${ }^{2}$

One part of system is also Social alarm, which can dial up responsible person in case of risky situation. This service is provided by digital carephone unit which offer to the user a high quality voice-to-voice connection in dangerous situation. It uses a standardized protocol called SIP which is a world standard protocol used to establish speech and video uplink via a broadband connection. In addition to the voiceto-voice alarm calls it provides communication layer between beneficiaries and carers for platform in MonAMI system.

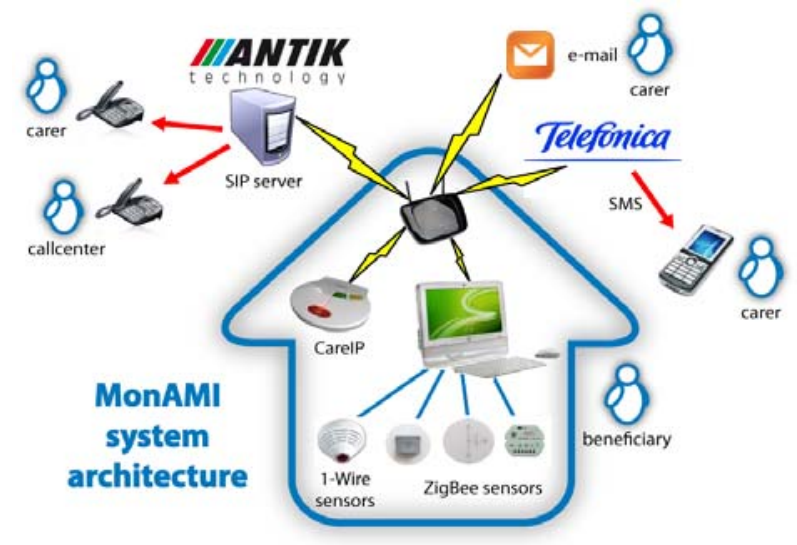

Fig.1. Architecture of used ICT system in senior's households for increasing their quality of life and safety

\section{ZigBee technology}

Reliability and usability are achieved by using different communication protocols and path ways.

ZigBee communication protocol is created by Zigbee alliance. Zigbee alliance is an association for companies working together to develop this standard. Zigbee technology can be installed for variety of applications such as smart houses, intelligent buildings and in industry ${ }^{3}$.Zigbee is based on IEEE 802.15.4 standard which defines physical and MAC layer for low cost and power for personal area network. The physical layer is working on three frequencies - 2, $4 \mathrm{GHz}$ in most countries, $868 \mathrm{MHz}$ in Europe and $915 \mathrm{MHz}$ in USA and Australia. All of these frequencies use DSSS (Direct sequence spread system) access and two types of modulations
B-PSK (binary phase shift modulation - for $2,4 \mathrm{GHz}$ ) and O-QPSK (offset - quadrature phase shift modulation - for 868 a $915 \mathrm{MHz}){ }^{4}$

ZigBee MAC Layer is defined in standard IEEE 802.15.4 and defines two types of devices (nodes): FFDs and RFDs. On FFD (fully functional device) is implemented full set of functions which are provided by MAC layer and can communicate with others FFDs and RFDs. One of the FFDs devices is always network coordinator. Coordinator sends beacons and provides synchronization and joins services. RFD (reduced functional device) acts like end device, it can only communicate with nearest FFD. ${ }^{4}$

ZigBee networks offers 3 types of topologies - star, mesh and tree. In star topology master-slave technology is used, where one off the FFDs is PAN coordinator = master. Other devices act like slaves (no matter if its RFD or FFD type of device). In mesh and tree topology is coordinator too and its function is to start network with initial parameters. ${ }^{5}$

ZigBee alliance created standard which defines higher levels of protocol. According to ${ }^{4}$ net-work layer provide services fort routing and "hop" network. On this level standard defines three types of devices FFD and RFD as end devices (compatible with IEEE 802.15.4) standard and Router. Router is FFD which provides services for routing. One FFD is coordinator and manage whole network. Interface between NWK (network layer) and the application layer (APL) is Application support sub-layer (APS). Application layer in general consists of three elements: APS sub layer, ZDO (ZigBee device object) and application objects designed by manufacturers. Application framework is the environment in which application objects are hosted on ZigBee devices - maximum 240 object can be defined. This system allows to objects discover each other and organize them in to a distributed network and also allows devices to connect or leave the network ${ }^{4}$.

\section{Wireless ZigBee sensors and actuators}

All chosen devices used in MonAMI platform to equip households of elderly are commercially available on the European market.

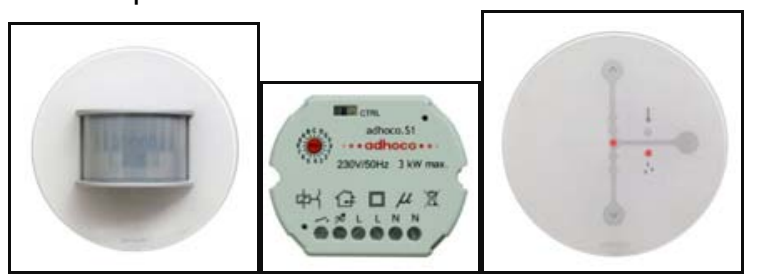

Fig.2. Wireless motion, luminance, temperature, humidity sensors and light actuator (in the middle) based on ZigBee technology

\section{The 1-wire technology}

1 -Wire $\AA$ products provide combinations of memory, mixed signal, and secure authentication functions via a patented single contact serial interface. With both 
power and communication delivered over the serial protocol, 1-Wire devices are unmatched in their ability to provide key functions to systems where interconnect must be minimized.

The 1-Wire $\circledR$ Net, sometimes known as a MicroLAN is a low-cost network based on a PC or microcontroller communicating digitally over twisted-pair cable with 1-Wire components. A 1-Wire Net based system consists of three main elements: a bus master with controlling software, the wiring and associated connectors and 1- Wire devices. The 1-Wire net allows tight control because no node is allowed to speak unless requested by the master, and no communication is allowed between slaves, except through the master.

The 1-Wire $®$ communication protocol can be generated with an 10 pin on a microprocessor; however, care must be taken to provide the correct timing and proper slew rates to create a reliable 1-Wire network. Uncontrolled slew rates can severely limit the length of a network and create sporadic behavior. ${ }^{6}$

\section{1-Wire sensors}

The 1-Wire sensors were mainly used due to stability of network during transmission of data. Second approach was dedicated to comparison of stability, reliability and adaptability of using the sensors. Wire network required more building changes (wires, adapters, new outlets and sockets) as wireless technology. It becomes easier to manipulate or restart in case of electricity outage. Wire technology also has to be constructed from base as prototypes designed to individuals. In contrast to wireless technology, sensors can be bought from market.

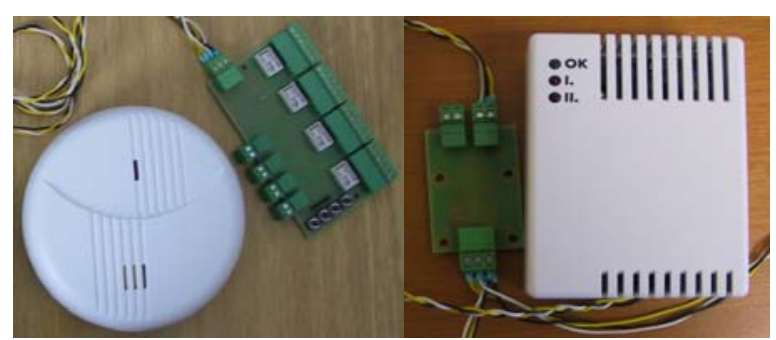

Fig.3. Gas and smoke sensors based on 1-wire technology

\section{OSGi platform and OSGi4AMI}

The main benefit of OSGi platform is modularity in Java programming language, which allows applications to be created from small, reusable and interoperable components. Each component, called bundle has its own life cycle (installation, start, update, stop, and uninstallation). A transition from one state to another is done without the need to restart the whole system. This significantly improved the development phase, where different sites were providing different application components and the whole implementation process was done in parallel. ${ }^{7}$
OSGi4AMI represents a reference point for all developers of the MonAMI system. It is a common interface framework developed as an open source technology (accessible at Source Forge) ${ }^{8}$, which enables through provided ontology the interaction between MonAMI Framework modules. This ontology defines the concepts used by MonAMI software modules of RG. The mechanism of the OSGi services layer enables registration, searching and notification functionality of new implemented services.

\section{TRIALS IN LABORATORY ENVIRONMENT}

The MonAMI platform and services were build and running at first in "Living labs" (prepared as fully functional household with typical rooms), where researchers designed and tested architecture, placement, devices and other parameters needed for installation in to real life. Afterwards, for services was created intuitive and interactive interface for interaction with users. Also interface for technician and careers to set up and individualize services were prepared. Laboratory is equipped with all developed interfaces, devices and actuators to show all possibilities of services for users which are interested in such type of services.

In our laboratory we are able demonstrate that developed services are technically feasible and usable for seniors and also show to users how these services can increase the quality of their life.

Users and their carers provided a good feedback to researchers especially in the content of services and user interface. Researchers collected their feedback in several stages of project period (1- before full testing in homes, during laboratory testing, 2- after first two weeks of testing at homes, and 3 - on the end of testing at homes).

Researchers have tested two types of communication in MonAMI. Wireless, based on ZigBee technology and wired, based on 1-Wire technology.

\section{TRIALS IN SENIOR'S HOUSEHOLDS}

Living Scale Field Trial was carried out in the homes of elderly and disabled people. Users were chosen in cooperation with House of Seniors. Selected users had to fulfill including criteria. These included age (seniors above 65 years), life situation (living alone) and health conditions (with disabilities: reduced mobility, memory problems, low vision, hearing problems). Finally 20 participants were choosed according these eligibility criteria. User's carers participated also in project trials and they provided feedback about their experiences with services during the trials.

Testing period started with pilot testing in two households. They have been equipped with Telco technology to achieve remote control of service installation from research centre. It enables installation of required services and also correction of failures or 
problems with services. After pilot was running correctly, testing started with installation into other households.

\section{Questionnaires and interviews}

During trial period data were gathered by questionnaires. Questionnaires were filled by users and also by careers. In the beginning, before LSFT trials started the Baseline structured questionnaire were completed. These interviews were focused on the social-demographical data and health status of user participated in LSFT.

In the middle of testing phase Mid-Term checkup questionnaires were applied. The mid-term face-toface interviews included filling the semi-structured questionnaires, where the first data about the accessibility, acceptability and suitability of the services were collected. The interviewer discussed each service enabled in the user's households. They evaluated satisfaction with each particular service, and questions how the services fitted into their daily lives. Also, the interfaces used in trial were discussed. The mid-term interviews were time demanding whereas each particular service installed in the user's household was discussed with both user and carer.

The post-trial instrumentation consisted primarily of in-depth, semi-structured interviews about users' perceptions of the benefits of the services. The research team visited each user again to perform faceto-face post-trial interview to collect detailed valuable data about the usefulness of the services and their contribution to the independence, health and wellbeing, social networking, safety-security, acceptability and e-inclusion. ${ }^{9}$

\section{Services used in LSFT}

Tested services were designed and based on daily needs of seniors and they represent modular system of services, with possibility install chosen services according the real needs and requests of end-user. These services are based mostly on if-then rules. The condition part combines information from several devices and technological services. The conclusion part is based on a combination of functional services and devices.

Services designed and developed in project were divided into three main categories according the aim of use.

Monitoring where beneficiary is able to monitor different parameters e.g. temperature, appliance usage, presence etc. locally or remotely. Monitoring is done through web based UI, so carer can also check if for example beneficiary is present at home if it is expected.

The Remote control is service mainly aimed for beneficiary; they could turn on/off lights or appliances. The lights are turned on automatically also in case of detected presence when the light threshold is below predefined threshold (this service is called LightSURE and for the majority beneficiaries is used in hall and toilet space).

Services for Alarm notification are dedicated to inform carer about various types of alarms e.g. smoke and gas leak danger, presence is detected in dangerous zone when not expected, social alarm etc.

All services are configurable and adaptable according the users' needs and the configuration and adaptation was made by technical staff during trials. The parameters for configuration purposes were gained from questionnaires filled with users before the start of trials.

Services used in household trials focused on user safety:

- GasSURE and SmokeSURE - services dedicated to monitoring dangerous level of smoke and gas concentration.

- AppSURE - switching off an electric device if this device is in usage for more than a predefined time or energy spent. It is useful when the user has forgotten to switch off e.g. iron, hotplate etc.

- ZoneSURE - if motion sensor recognizes /do not recognizes a user activity during a predefined time in predefined area, responsible person is informed about this situation.

- TempSURE - sends an alert or trigger an alarm if temperature is for longer time lower or higher then predefined threshold.

Not all services were installed in each household. Before installation of the system some beneficiaries refused particular services, because they considered them unnecessary or they required hard installation in their living environment.

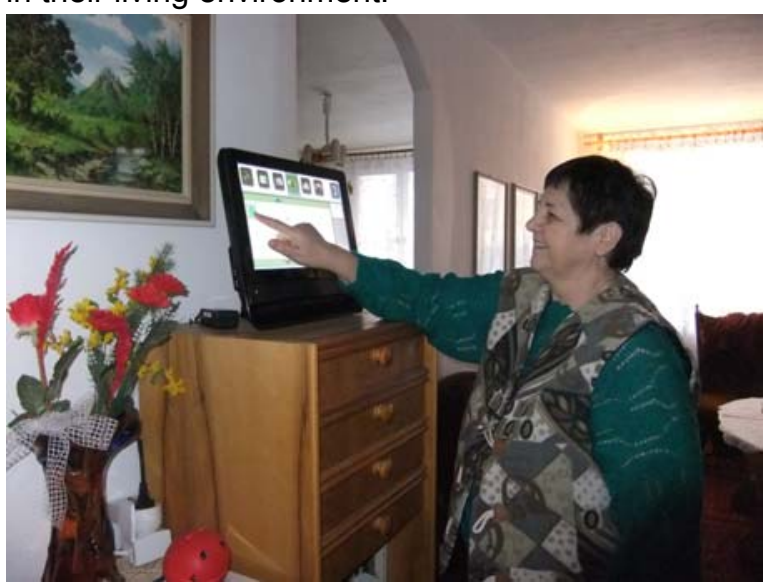

Fig.4. User in LSFT trials

\section{RESULTS AND DISCUSSION}

Testing was done in three countries in Europe (Sweden, Spain, and Slovakia) due to comparison in cultural, political and legislative issues. We installed and tested services in 20 households located in the area 
of Košice city in Slovakia for seniors living there alone.

During trials in households several technical problems occurred from the beginning. The information from social alarm was sent to informal carers, mainly family relatives. The CareIP social alarm had problem with SIP server on the side of internet provider.. The communication was canceled after 20 seconds. To solve the issue we had changed SIP implementation and server due to specific condition. In some households happened that users turned off the system or disconnect devices. Intentional shutdown was caused because they were unsatisfied with one particular service and this was for them the fastest way to solve their problem or the unintentional shutdown of the system was often caused by accidental removal of the RG plug.

SmokeSURE was mostly appreciated safety service with 5 successful uses while testing. Only once time false alarm was recognized. The false alarm was caused by fault of device which was replaced by new ones.

Configuration of user interface was underestimated from our side, since the carers evaluated it as rather complex and should be improved in future. Because of not so high computer literacy of middle-age people in Slovakia, and this group created the largest number of unformal carers - children of beneficiaries.

Based on Post-Trial questionnaire we can conclude that users were satisfied with choosen services because they felt safer and also carers were satisfied. Users evaluated as the most useful and necessary services ZoneSURE, ConnectSURE (social alarm, which connects the user to carer through CareIP, who could remotely check how dangerous the situation is) and AmiPAL a reminder of the events like taking pills, visiting a doctor, etc.

On the other hand they wanted also other services, which were not included in project like video doorman.

Comparison between wire and wireless technology brings remarkable notices. Wireless technology is reliable and more adaptable for using in regular senior's household. Sensors can be mounted just by placing on specified spot, in case of outage or restart they easily find way to connect master slave or network coordinator. All these positives are underlined with low electricity consumption.

The experience from the whole trial is very helpful in future development of such services in our region. MonAMI field trial opens discussions with local stakeholders leading to formulation of the new tasks in social area like demanding the creation of an effective platform for the Social alarm service in Košice city. Activities within the frame of the project has pushed the Municipality of Košice to facilitate the discussions about the care for elderly and disabled based on ICT.

Project and results from trials with seniors accelerated cooperation between local partners and stakeholders to prepare other solutions and start to implement these solutions into business. From the user's point of view, they were satisfied mainly in the area of safety and possibility to be monitored in secure and unobtrusive way and also they highly appreciated to manage contact with other people, children or seniors.

\section{ACKNOWLEDGMENT}

The work is part of the solution within the international donation 6th EC Framework Program - Priority 2.3.2.10 e-Inclusion „Mainstreaming on Ambient Intelligence "IST-5-0535147 and Slovak grant agency VEGA „1/1162/11 -- Teoretické princípy, metódy a prostriedky diagnostiky a rehabilitácie mobility seniorov".

\section{References}

1. Kung A., Jean-Bart B., "Making AAL Platforms a Reality," Ami-10. International conference on ambient intelligence, Malaga, Spain, 2010.

2. URC Consortium, "MyURC - Index of Technical Reports" available on http://myurc.org/

3. Hetherington, W., "Intelligent Building Concept" EMCS Engineering, Ontario, 1999

4. ZigBee Alliance, "ZigBee specification", available on http://www.zigbee.org/Specifications/ZigBee/downlo ad.aspx

5. Pillai P., Chook V.W.C., Chessa S., Gotta P. B. A., Fun $\mathrm{Hu}$ Y., "Wireless sensor networks: A survey on the state of the art and the 802.15.4 and ZigBee standards", Computer Communications, Vol. 30(7), pp.1655-1695, 2007.

6. Šimšík D., et al., "Wireless and 1-wire solutions for inclusive home environment of seniors", In: AAATE 2009, Assistive Technology Research Series, Vol. 25, pp. 303-308, 2009.

7. Šimšík, D., et al., "MonAMI platform, trials and results", 10th IEEE Jubilee International Symposium on Applied Machine Intelligence and Informatics, Herlany, 2012.

8. OSGi4AMI,"Sourceforge". http://sourceforge.net/projects/osgi4ami/

9. Siman, D., et. al., "Increasing of the quality of life of seniors by automation of households and application of the services based on ICT in Slovakia", Trends in biomedical engineering 2011, vol.9, pp. 67-69, 2011. 